\title{
Overflow Microfluidic Networks for Open and Closed Cell Cultures on Chip
}

 \\ Emmanuel Delamarche*,t
}

IBM Research-Zurich, Säumerstrasse 4, 8803 Rüschlikon, Switzerland, Neuro-Zone s.r.l., via Fratelli Cervi 93,
20090 Segrate, Italy, Department of Pharmacology, CNR Institute of Neuroscience, University of Milano, via Vanvitelli
32, 20129 Milano, Italy, and Fondazione Filarete, viale Ortles 22/4, 20139 Milano, Italy

Microfluidics have a huge potential in biomedical research, in particular for studying interactions among cell populations that are involved in complex diseases. Here, we present "overflow" microfluidic networks (oMFNs) for depositing, culturing, and studying cell populations, which are plated in a few microliters of cell suspensions in one or several open cell chambers inside the chip and subsequently cultured for several days in vitro (DIV). After the cells have developed their phenotype, the oMFN is closed with a lid bearing microfluidic connections. The salient features of the chips are (1) overflow zones around the cell chambers for drawing excess liquid by capillarity from the chamber during sealing the oMFN with the lid, (2) flow paths from peripheral pumps to cell chambers and between cell chambers for interactive flow control, (3) transparent cell chambers coated with cell adhesion molecules, and (4) the possibility to remove the lid for staining and visualizing the cells after, for example, fixation. Here, we use a two-chamber oMFN to show the activation of purinergic receptors in microglia grown in one chamber, upon release of adenosine triphosphate (ATP) from astrocytes that are grown in another chamber and challenged with glutamate. These data validate oMFNs as being particularly relevant for studying primary cells and dissecting the specific intercellular pathways involved in neurodegenerative and neuroinflammatory brain diseases.

Microfluidics are emerging as invaluable tools for research on cells for a number of reasons. ${ }^{1-3}$ First, they enable experiments on cells to be performed with well-defined chemical and topographical environments. ${ }^{4-9}$ Second, they can reduce the number

\footnotetext{
* To whom correspondence should be addressed. E-mail: emd@ zurich.ibm.com.

${ }^{\dagger}$ IBM Research.

* Neuro-Zone s.r.l.

$\S$ CNR Institute of Neuroscience.

"Fondazione Filarete.

(1) Persidis, A. Nat. Biotechnol. 1998, 16, 488-489.

(2) Folch, A.; Toner, M. Annu. Rev. Biomed. Eng. 2000, 2, 227-256.

(3) Prokop, A.; Prokop, Z.; Schaffer, D.; Kozlov, E.; Wikswo, J.; Cliffel, D.; Baudenbacher, F. Biomed. Microdevices 2004, 4, 325-339.

(4) Takayama, S.; Ostuni, E.; LeDuc, P.; Naruse, K.; Ingber, D. E.; Whitesides, G. M. Nature 2001, 411, 1016.

(5) Meyvantsson, I.; Beebe, D. J. Annu. Rev. Anal. Chem. 2008, 1, 423-444.

(6) Breslauer, D. N.; Lee, P. J.; Lee, L. P. Mol. BioSyst. 2006, 2, 97-112.
}

of cells needed for experiments. ${ }^{1}$ Third, they can be used for experiments in which a precise number of cells can be stimulated and observed accurately. ${ }^{10-12}$ Last, they permit experiments to be performed in parallel and/or with high throughput. ${ }^{13}$ Despite these possibilities, microfluidics are not well established for daily research work on cells, as they lack user friendliness and require specific and often cumbersome methods for depositing and culturing cells. This is further complicated when taking into consideration more relevant cell systems such as primary cells, ${ }^{14,15}$ which may require longer culturing times before being ready for specific assays (i.e., primary neurons need to be cultured several days in vitro (DIV) before establishing mature synaptic contacts). Although progress on sealing a Petri dish with a hybrid microfluidic-vacuum platform was recently demonstrated by Chung et al., ${ }^{9}$ interfacing "open" cell cultures with microfluidics for cell cultures that are only a few microliters in volume remains a great challenge. Similarly, depositing cells on a treated glass slide and gluing a lid having ports onto the slide provides a method for enclosing living cells inside a microfluidic flow path. ${ }^{16}$ Such a method would be hard to implement with multiple types of cells and can be simplified if the gluing step could be omitted. Finally, Shuler et al. developed powerful micro cell culture analogs to assess the cytotoxicity of drugs on cells. ${ }^{17}$ In this work, cells were mixed with a gel at a temperature of $4{ }^{\circ} \mathrm{C}$, then added to the micro cell culture analogs, and entrapped in the gel by raising its temperature to $37{ }^{\circ} \mathrm{C}$. Using a low temperature (especially if primary cells are used) as well as challenges with air bubbles

(7) Figallo, E.; Cannizzaro, C.; Gerecht, S.; Burdick, J. A.; Langer, R.; Elvassore, N.; Vunjak-Novakovic, V. Lab Chip 2007, 7, 710-719.

(8) El-Ali, J.; Sorger, P. K.; Jensen, K. F. Nature 2006, 442, 403-411.

(9) Chung, B. G.; Park, J. W.; Hu, J. S.; Huang, C.; Monuki, E. S.; Jeon, N. L. BMC Biotechnol. 2007, 7, 60.

(10) Taylor, A. M.; Rhee, S. W.; Tu, C. H.; Cribbs, D. H.; Cotman, C. W.; Jeon, N. L. Langmuir 2003, 19, 1551-1556.

(11) Lovchik, R. D.; Bianco, F.; Matteoli, M.; Delamarche, E. Lab Chip 2009, 9, 1395-1402.

(12) Lucchetta, E. M.; Lee, J. H.; Fu, L. A.; Patel, N. H.; Ismagilov, R. F. Nature 2005, 434, 1134-1138.

(13) Faley, S.; Seale, K.; Hughey, J.; Schaffer, D. K.; VanCompernolle, S.; McKinney, B.; Baudenbacher, F.; Unumatz, D.; Wikswo, J. P. Lab Chip 2008, 8, 1700-1712.

(14) Rhee, S. W.; Taylor, A. M.; Tu, C. H.; Cribbs, D. H.; Cotman, C. W.; Jeon, L. N. Lab Chip 2005, 5, 102-107.

(15) Millet, L. J.; Stewart, M. E.; Sweedler, J. V.; Nuzzo, R. G.; Gilette, M. U. Lab Chip 2007, 7, 987-994.

(16) Morel, M.; Bartolo, D.; Galas, J.-C.; Dahan, M.; Studer, V. Lab Chip 2009, 9, 1011-1013.

(17) Sung, J. H.; Shuler, M. L. Lab Chip 2009, 9, 1385-1394. 




Figure 1. Different types of cells in the brain. (a) Section of a rat brain tissue, stained with fluorescence dyes to visualize key cells and structures. Reprinted with permission from ref 18. Copyright 2008 Elsevier B.V. (b) Simplified illustration of the complex interactions between some of the cell populations involved in neurodegenerative diseases ( $\mathrm{N}$ : neurons, $\mathrm{A}$ : astrocytes, M: microglia, O: oligodendrocytes, C: blood capillary).

and leaks when screwing a cover on the micro cell culture analogs also entails technical challenges.

\section{CELLULAR PATHWAYS IN MICROFLUIDICS}

In addition to the above-mentioned advantages and drawbacks of microfluidics when working with cells, entirely new types of experiments become possible when distinct cell populations are placed within a microfluidic device, which allows one to simultaneously carry out a functional analysis on stimulated versus control cells in a miniaturized, highly controlled microenvironment. Besides enabling the analysis of cellular behaviors, such as cell differentiation, motility, or response to external trophic or toxic stimuli, microfluidics open the possibility of dissecting cell-to-cell communication in complex intercellular scenarios, such as those represented by the nervous or the immune systems, where the deep comprehension of molecular interchanges among different cell types (i.e., in the central nervous system, Figure 1) is crucial for the correct understanding of the mechanisms involved in the onset of diseases. In the brain, this is particularly true for the neuroinflammatory events which contribute to neuronal death in neurodegenerative diseases. The latter are among the most devastating diseases, which account for $30 \%$ of healthcare costs in industrialized countries and an estimated 35\% of the disease burden within the seven major pharma markets. Notably, today there are no drugs available that can arrest or reverse neurodegeneration.

(18) Bjornsson, C. S.; Lin, G.; Al-Kofahi, Y.; Narayanaswamy, A.; Smith, K. L.; Shain, W.; Roysam, B. L. Neurosci. Methods 2008, 170, 165-178.
In the last few years, it has become clear that dysfunctions of the synapse (the functional contact between neurons) are central to the etiology and progression of a wide range of neurological and psychiatric disorders, including neurodegenerative diseases, schizophrenia, autism, depression, and many others, which can therefore be collectively regarded as synaptopathies. Besides specific defects in neuronal proteins, activation of immune mechanisms and inflammation play a crucial role in synaptopathies. Bidirectional functional interactions among neurons, astrocytes, and microglia, through the release of soluble chemical mediators, govern both the sequence of inflammatory events (cascades of inflammatory mediators) and the pathological outcome (damage or absence of damage to the neurons). However, the pathways of these inflammatory signaling cascades remain cryptic, the major mediators unknown, and the sequential flux of molecular information among the different cell types still undefined. The scientific community is currently facing the challenge of dissecting the neuroinflammatory cascades among microglia, astrocytes, and neurons by breaking down the cellular networks and controlling the flow of inflammatory mediators among these cells. This is crucial for understanding the molecular mechanisms that are at the basis of neuronal damage, in order to define smarter strategies for tackling neurodegenerative processes.

\section{CONCEPT OF OVERFLOW MICROFLUIDIC NETWORKS}

Here, we describe a concept for microfluidics in which cells are plated and cultured in an open part of the chip and studied under "microfluidic conditions" after sealing the open chip with a lid. Plating and culturing the cells with this chip is extremely simple: the surface of the chip is treated with cell-adhesion molecules such as fibronectin or poly-L-lysine; then, a few microliters of cell suspension and medium are added to the open chip, and the open chip is placed in a cell incubator to provide cells with the appropriate temperature and level of $\mathrm{CO}_{2}$ to enable development of their phenotype. Whenever needed, the culture medium is changed by pipetting. In this way, cells can be maintained for any duration of time akin to cells cultured in T-flasks. We first illustrate how to deposit one type of cell on a chip and then demonstrate that two types of primary cells can be deposited on a chip and a pathway between the cells established.

We call the open chip an overflow microfluidic network (oMFN), the design of which is shown in Figure 2a. The oMFN is molded in poly (dimethylsiloxane) (PDMS), $150 \mu \mathrm{m}$ deep, and possesses a central cell chamber having a volume of $\sim 0.5 \mu \mathrm{L}$. The chamber is connected to inlet and outlet microchannels via flow-distributing structures. ${ }^{11}$ The central features of the oMFN are the "overflow" zones containing capillary microstructures, ${ }^{19,20}$ which are adjacent to the cell chamber.

After cells had sufficient time to grow and develop their phenotype in the cell chamber, a Si lid having vias $(800 \mu \mathrm{m}$ in diameter) and fittings is placed by hand over the oMFN using alignment marks as visual aids. The lid $(4-15 \mathrm{~g}$ in weight, depending on the type of ports and fittings used) is approached

(19) Juncker, D.; Schmid, H.; Drechsler, U.; Wolf, H.; Wolf, M.; Michel, B.; de Rooij, N.; Delamarche, E. Anal. Chem. 2002, 74, 6139-6144.

(20) Zimmermann, M.; Schmid, H.; Hunziker, P.; Delamarche, E. Lab Chip 2007, 7, 119-125. 




Figure 2. Design ( $a$ and $b$ ) and photographs of a PDMS oMFN before (c) and after (d) assembly with the Si lid bearing ports, fittings, and tubings.

slowly until it touches the droplet of cell suspension located in the cell chamber. It is then allowed to come into contact with the oMFN under its own weight. Sometimes, gently pressing the middle of the lid using tweezers is necessary to trigger contact between the oMFN and the lid. During this step, excess liquid in the cell chamber flows over the chamber sidewall and connects with the overflow areas, Figure $2 \mathrm{~b}$. The liquid is pulled away from the chamber owing to the capillary pressure generated by the microstructures arrayed in the overflow areas, and the lid conforms to the elastomeric oMFN. Not having the overflow areas results in having a thin film of liquid squeezed between the MFN and the lid, which makes the surfaces slide against each other unpredictably during assembly and the interstitial liquid will fill remote microfluidic structures, thereby entrapping air bubbles. The oblong microstructures visible in Figure $2 \mathrm{~b}$ have a length of $100 \mu \mathrm{m}$, a width of $60 \mu \mathrm{m}$, an intrarow spacing of $60 \mu \mathrm{m}$, and interrow spacing of $30 \mu \mathrm{m}$. These structures and their lattice were selected from earlier work on capillary pumps for microfluidic diagnostic chips..$^{20,21}$ Excess liquid quickly fills the overflow zone with a straight filling front, thereby preventing air entrapment in the overflow area and pushing air toward the periphery of the oMFN. One overflow area can accommodate $4.35 \mu \mathrm{L}$ of liquid; by connecting these areas to the alignment marks using microchannels, venting and draining of the overflow areas are achieved. Figure 2c,d, respectively, shows a photograph of a PDMS oMFN and of an oMFN sealed with a Si lid having ports. Si was selected as a material for the lid because of its mechanical stability, flatness (the face of the Si wafer in contact with PDMS is polished), chemical resistance, and compatibility with adhesives such as those used for bonding ports. The footprint of the ports and tubings, visible in Figure 2d, can be reduced by soldering metallic wires directly to the lid if needed. ${ }^{22}$

\section{EXPERIMENTAL SECTION}

Chemicals, Biochemicals, and Buffers. Fibronectin from human plasma, poly-L-lysine, Trypan blue, trypsin, ethylenedi-

(21) Gervais, L.; Delamarche, E. Lab Chip 2009, 9, 3330-3337.

(22) Murphy, E. R.; Inoue, T.; Sahoo, H. R.; Zaborenko, N.; Jensen, K. F. Lab Chip 2007, 7, 1309-1314. aminetetraacetic acid (EDTA), and phosphate buffered saline (PBS) were purchased from Sigma Aldrich (Buchs, Switzerland). Yo-Pro 1 dye, NBD, and Falloidin T-red (1:200) were purchased from Invitrogen (Milano, Italy). Beta3 tubulin monoclonal antibodies (1:400, Promega, Italy), synaptotagmin (1:100), and v-Glut1 antibodies (1:100) were purchased from Synaptic Systems (Gottingen Germany); monoclonal anti-glial fibrillary acidic protein produced in mouse (GFAP) (1:400) was from Sigma, and ionized calcium binding adaptor molecule 1 antibody from rabbit (IBA) (1:100) was from Duotech. Purified water was produced using a Simplicity 185 system from Millipore (Zug, Switzerland).

Fabrication of the PDMS oMFNs and Si Lids. The oMFNs were molded in PDMS using Si molds made from 4-in. Si wafers (Siltronix, Geneva, Switzerland). The microfabrication of the molds was done using standard photolithography and photoplotted polymer masks (64'000 dpi, Zitzmann GmbH, Eching, Germany) and deep reactive ion etching (STS ICP, Surface Technology Systems, Newport, UK). The depth of the structures was $150 \mu \mathrm{m}$, and $\sim 5 \mathrm{~nm}$ of a fluorinated material was deposited at the end of the etching process to act as an antiadhesive layer. Molding the oMFNs was done by pouring Sylgard 184 prepolymers (Dow Corning, Midland, MI) onto a Si mold that was placed in a clean Petri dish. The ratio of polymer to curing agent was 10:1. After a polymerization time of at least $12 \mathrm{~h}$ at $60^{\circ} \mathrm{C}$, the PDMS oMFNs were peeled off the mold and separated using a scalpel. The oMFNs were stored in a clean Petri dish with the structures in contact with the bottom of the dish until they were used for experiments.

The Si lids were fabricated using the same method and equipment than that for the $\mathrm{Si}$ molds. The vias and alignment marks were etched through a $500 \mu \mathrm{m}$ thick 4 in. Si wafer, and Nanoport Assemblies from Upchurch Scientific (Ercatech, Bern, Switzerland) were centered over the vias and glued to the backside of the Si lids.

Peripheral Equipment. Tubes and standard fittings were purchased from Upchurch Scientific. High precision motorized syringe pumps (Cetoni GmbH, Korbussen, Germany) were used to move liquids through closed oMFNs and were equipped with $50 \mu \mathrm{L}$ syringes from Hamilton (Bonaduz, Switzerland). The closed oMFNs were placed onto the stage on an inverted microscope (Nikon Eclipse TE300, Egg, Switzerland) for perfusion with medium and staining procedures. The microscope was equipped with a camcorder (Sony CDR SR100E, Schlieren, Switzerland). Some images were taken using an upright microscope (Eclipse 90i, Egg, Switzerland) equipped with a color CCD camera (Nikon DXM 1200C, Egg, Switzerland). Closing procedures with colored liquids were recorded with the camcorder mounted to a Leica stereomicroscope. The confocal images from open oMFNs were acquired using a LSM 510 Meta confocal microscope (Zeiss, Germany) and a Nikon (Tokyo, Japan) 20x objective with a sequential setting.

Cell Deposition, Cultivation, and Staining. Depending on the cells used for the experiment, the oMFNs were coated with fibronectin or poly L-lysine. Fibronectin coating was done by covering an oMFN with a sterile solution of $50 \mu \mathrm{g} \mathrm{mL}^{-1}$ fibronectin in PBS for 30 min at room temperature. The oMFN was then rinsed with PBS and water and dried under a stream 
of $\mathrm{N}_{2}$. Coating an oMFN with poly L-lysine required a brief plasma treatment of the oMFN. This was done in a plasma generator (Technics Plasma 100-E, Florence, KY) in an air plasma at $200 \mathrm{~W}$ (coil power) for $30 \mathrm{~s}$. Directly after the plasma treatment, the oMFN was coated with a $0.5 \mathrm{mg} \mathrm{mL}^{-1}$ solution of poly L-lysine in a borate buffer and incubated at room temperature overnight.

Cell deposition was done by placing a $5 \mu \mathrm{L}$ drop of fresh medium onto the chamber of a prepared oMFN and pipetting 2 $\mu \mathrm{L}$ of cell suspension (ca. 1500 cells) into the drop of medium. The cells sedimented to the bottom of the chamber and were allowed to attach to the surface by placing the oMFNs in a cell incubator $\left(37{ }^{\circ} \mathrm{C}, 5 \% \mathrm{CO}_{2}\right)$. To avoid evaporation of the small drops, $100 \%$ humidity was maintained by placing some water near the oMFNs located in Petri dishes. Primary cells required an exchange of medium after $24 \mathrm{~h}$.

HeLa cells were cultivated in DMEM medium. The cell suspension used for seeding in oMFNs contained $\sim 10^{5}$ cells $\mathrm{mL}^{-1}$. After 1 day of incubation, the oMFN was closed and perfused with fresh medium at a flow rate of $2 \mu \mathrm{L} \mathrm{min}^{-1}$ for $5 \mathrm{~min}$. The cells were exposed for 2 min to a Trypan Blue solution perfusing the chamber at a flow rate of $2 \mu \mathrm{L} \mathrm{min}{ }^{-1}$. After flushing the cells with fresh medium for another $2 \mathrm{~min}$, images were taken and the viability of the cells checked using an upright microscope.

SY5Y neuroblastoma were obtained as a kind gift from Dr. Fornasari (CNR Institute of Neuroscience, Milano Italy) and cultivated in RPMI medium (Sigma, Italy) supplemented with 10\% fetal bovine serum (Invitrogen, Italy), 1\% penicillin/streptomycin (Invitrogen Italy), and $1 \% \mathrm{~L}$-glutamine (Invitrogen, Italy) at $37^{\circ} \mathrm{C}$ and $5 \% \mathrm{CO}_{2}$. The cell suspension used for seeding contained $\sim 10^{5}$ cells $\mathrm{mL}^{-1}$. After 1 day of incubation, the oMFN was closed and perfused with fresh medium at a flow rate of $2 \mu \mathrm{L}$ $\min ^{-1}$ for $5 \mathrm{~min}$. The cells were then exposed for $2 \mathrm{~min}$ to a 1 $\mathrm{mM}$ solution of FM 143 (Sigma-Aldrich, Italy) by perfusing the chamber at a flow rate of $2 \mu \mathrm{L} \mathrm{min}^{-1}$. After washing the cells with fresh medium for another $2 \mathrm{~min}$ at the same flow rate, images were taken using an upright microscope.

Primary neuronal cultures were prepared from embryonic (E18) rat hippocampus (Sprague Dowley rats, Charles River, Calco, Italy) as previously described. ${ }^{23}$ The medium was exchanged after 1 day of incubation. The oMFN was closed after 6 days, and fresh medium perfused at a flow rate of $2 \mu \mathrm{L} \mathrm{min}^{-1}$ for $5 \mathrm{~min}$. The cells were exposed for $2 \mathrm{~min}$ to a $1 \mathrm{mM}$ solution of FM 143 by perfusing the chamber at a flow rate of $2 \mu \mathrm{L} \mathrm{min}^{-1}$. After washing the cells with fresh medium for another $2 \mathrm{~min}$ at the same flow rate, images were taken using an upright microscope.

Primary astrocytes and microglia cortical mixed-glia cultures from rat pups (P2) were obtained using previously described methods ${ }^{24}$ Briefly, after dissection, the cortices were dissociated by treatment with trypsin $\left(0.25 \%\right.$ for $10 \mathrm{~min}$ at $\left.37^{\circ} \mathrm{C}\right)$ followed by fragmentation with a fire-polished Pasteur pipet. The dissociated cells were plated onto PDMS oMFNs $(\sim 2000$ cells per chamber), and the cultures were grown in Minimum

(23) Verderio, C.; Coco, S.; Bacci, A.; Rossetto, O.; De Camilli, P.; Montecucco, C.; Matteoli, M. I. Neurosci. 1999, 19, 6723-32.

(24) Calegari, F.; Coco, S.; Taverna, E.; Bassetti, M.; Verderio, C.; Corradi, N.; Matteoli, M.; Rosa, P. L. Biol. Chem. 1999, 274, 22539-22547.

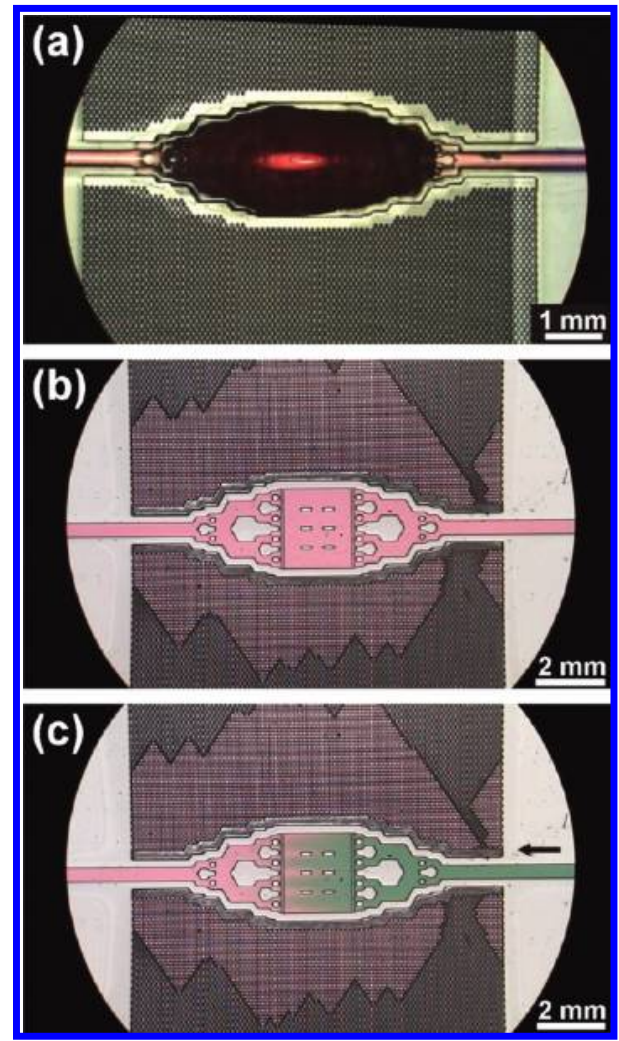

Figure 3. Frames from a video showing (a) the assembly of a Si lid onto an oMFN containing $\sim 5 \mu \mathrm{L}$ of red-colored water, (b) the sealed chip with water distributed inside the microfluidic flow path and in some of the overflow areas, and (c) the exchange of the red-colored water by a green-colored water by pumping liquid through one port of the sealed chip.

Essential Medium (Invitrogen, Italy) supplemented with 10\% horse serum (Euroclone Ltd., UK) and glucose at a final concentration of $5.5 \mathrm{~g} \mathrm{~L}^{-1}$. Primary microglia were obtained by shaking the mixed glial cultures 4 times at $150 \mathrm{rpm}$ for 5 min with 2 min pauses in between.

Immunofluorescence: Cells were fixed for $15 \mathrm{~min}$ at room temperature with $4 \%$ paraformaldehyde in $0.12 \mathrm{M}$ phosphate buffer containing $0.12 \mathrm{M}$ sucrose. The fixed cells were detergentpermeabilized and labeled with primary antibodies followed by fluorescently labeled conjugated secondary antibodies. The oMFNs were placed in $70 \%$ glycerol in PBS containing $1 \mathrm{mg} \mathrm{mL}^{-1}$ phenylendiamine. The images were acquired using a BioRad MRC-1024 confocal microscope equipped with the LaserSharp 3.2 software.

\section{RESULTS AND DISCUSSIONS}

Overflow Microfluidic Network for One Cell Population. Figure 3 shows the sealing of an oMFN, the cell chamber of which was covered with $\sim 5 \mu \mathrm{L}$ of water colored in red. The ports on the lids were connected via tubings to a reservoir (inlet port) and a high-precision computer-controlled pump (outlet port, Cetoni GmbH, Korbussen, Germany). The ports and tubings were filled with water prior to assembling the lid and oMFN to prevent the trapping of air in the microfluidic flow paths. Positioning the lid and having all excess liquid moved to the overflow areas took 5-10 s. After assembly, the liquid 


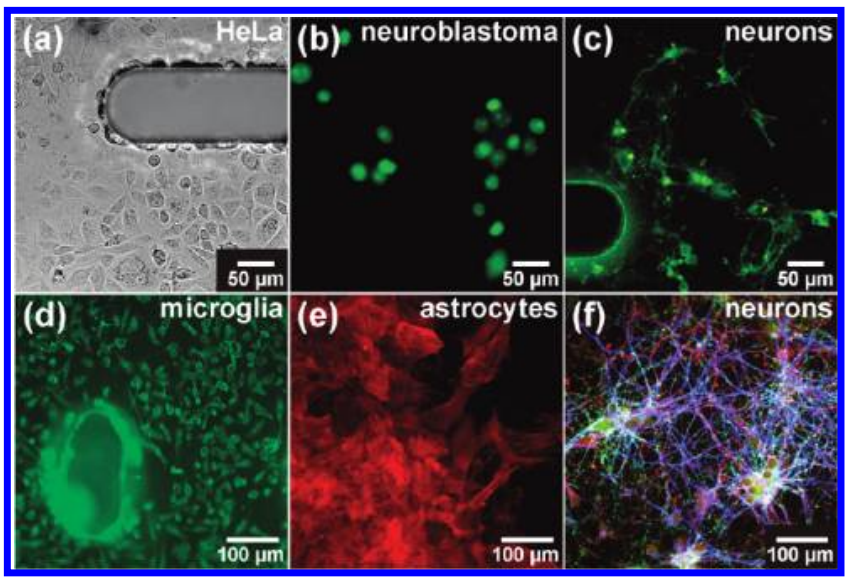

Figure 4. Brightfield and fluorescence microscope images of various types of cells cultured in oMFNs for 1 DIV (a, b, and d) up to 6 DIV (c, e, and f).

(colored in green) was pumped into the assembled microfluidic chip at a flow rate of $2 \mu \mathrm{L} \mathrm{min}^{-1}$. Liquid was pulled through the chamber to create a negative pressure in the oMFN, and flow rates between 1 and $3 \mu \mathrm{L} \mathrm{min}^{-1}$ were typically used. Prior to depositing cells, PDMS oMFNs were treated with a solution of polylysine (neurons) or fibronectin (other cells). PDMS oMFNs were exposed for $30 \mathrm{~s}$ to an $\mathrm{O}_{2}$-based plasma to oxidize their surface and create the negative charges needed to immobilize polylysine via electrostatic interactions.

Cell lines (HeLa and SY5Y neuroblastoma) and primary cells from the central nervous system (microglia, astrocytes, and neurons) were deposited in oMFNs. First, $5 \mu \mathrm{L}$ of culture medium was placed on the cell chamber, and then, $2 \mu \mathrm{L}$ of cell suspension was pipetted into the medium. Cells reached the surface of the chamber by sedimentation and were allowed to attach. Incubation of the cells proceeded for 1 (HeLa, SY5Y) to 6 DIV (primary cells). The oMFN was then closed as described above, and the morphology of cells and functionality of the sealed oMFNs were tested by staining the cells using vital dyes and using an inverted microscope. Figure $4 \mathrm{a}$ is a brightfield image of HeLa cells in the closed chamber. Images in Figure $4 \mathrm{~b}, \mathrm{c}$ were taken after cell membrane live staining in the closed microfluidic system. The images in Figure $4 \mathrm{a}-\mathrm{c}$ show that cells grow normally in PDMS chambers. Figures $4 \mathrm{~d}-\mathrm{f}$ are fluorescence microscope images of primary cells fixed and retrospectively labeled with cell-specific marker to show that primary brain cells attach and grow normally on oMFNs. If needed, the lid can also be made from glass ${ }^{25} \mathrm{so}$ as to visualize cells using an upright microscope or differential interference contrast microscopy. We were not able to close and remove the lid repeatedly without compromising the viability of the cells. We think that the shear stress exerted on the cells by the liquid pulled with the cover and/or the small volume of liquid $(<1 \mu \mathrm{L})$ left in the chamber account for this. Separating the lid from the oMFN, while pumping medium into one port and having the second port closed, might solve this problem.

Overflow Microfluidic Network for Two Cell Populations. The concept of the oMFN is here extended to a chip having two cell chambers. Figure 5 a shows the photograph of a two-chamber oMFN assembled with a Si lid having 6 ports for fluidic connec-

(25) Madou, M. Fundamentals of Microfabrication; CRC Press: Boca Raton, FL, 2002 . tion. The oMFN has a footprint of $32 \times 26 \mathrm{~mm}^{2}$, and its two cell chambers are only $3 \mathrm{~mm}$ apart to enable visualization of both chambers simultaneously using a $4 \times$ microscope objective. The layout of the channels and corresponding ports allows liquids to be drawn sequentially, or independently if needed, through the chambers. ${ }^{26}$ The entire overflow zone can accommodate up to $48 \mu \mathrm{L}$ of excess liquid. Typically, $\sim 7 \mu \mathrm{L}$ of cell suspension is used to plate and culture the cells in each chamber. Then, the chip is closed with the lid, and cell culture medium is pumped through the chambers using ports 1 (inlet) and 6 (outlet).

A known biochemical pathway of intercellular communication between primary astrocytes and microglia was performed to validate the system: primary cortical astrocytes in the first chamber were exposed to $50 \mu \mathrm{M}$ glutamate, a condition which is known to induce the regulated release of adenosine triphosphate (ATP). Upon transfer into the second chamber, the gliotransmitter activated purinergic receptors on microglia cells. For these experiments, astrocytes were cultivated in the left cell chamber for $3 \mathrm{DIV}$, while microglia were plated in the right chamber for 1 DIV. The oMFN was then closed using the lid, and regular morphology of the plated cells was assessed by inverted microscope observation. A growth medium was perfused for $\sim 3 \mathrm{~min}$ at a flow rate of $1 \mu \mathrm{L} \mathrm{min}^{-1}$ from port 1 to 6 before introducing a buffered solution containing $50 \mu \mathrm{M}$ glutamate and $20 \mu \mathrm{g} \mathrm{mL}$ propidium iodide (PI) dye for $20 \mathrm{~min}$ at a flow rate of $1 \mu \mathrm{L}$ $\mathrm{min}^{-1}$. It is well-known that glutamate-induced release of ATP results in activation of purinergic receptors on microglia cells and, in particular, activation of ionotropic P2X7 receptor. ${ }^{27}$ Prolonged activation of P2X7 is known to induce the formation of an aspecific pore, measured by dye uptake, which is known to induce sustained intracellular calcium levels in microglia leading to the release of neuroinflammatory mediators. ${ }^{28} \mathrm{In}$ our experiment, we show that ATP conveying in the second chamber as a consequence of astrocyte stimulation with glutamate leads to an uptake of PI by microglia. The micrographs in Figure $5 b-g$ show images (10× objective) of microglia after three independent experiments. Microglial dye uptake in Figure $5 \mathrm{c}$ indicates cell exposure to ATP, which was released by astrocytes and convected to the second chamber. Direct PI uptake by the microglia following glutamate exposure is excluded because omitting the astrocytes in the first chamber did not lead to staining of the microglia by PI, Figure 5e. As an additional control experiment, the astrocytes were stimulated with glutamate, this time having an addition of oxidized ATP (oATP, $100 \mu \mathrm{g} \mathrm{mL} \mathrm{m}^{-1}$ ) to the glutamate/PI solution. Oxidized ATP inhibits P2X7 receptor of microglia, and, as expected, a strong reduction of dye uptake by microglia is observed (compare Figure $5 \mathrm{~g}$ with Figure $5 \mathrm{c}$ ). Figure $5 \mathrm{~h}$ shows the percentage of PI positive cells relative to the number of cells observed in brightfield for the two experiments (with and without receptor inhibition). Moreover, the oMFN is not permanently bonded to the Si lid, so cells can be fixed after the experiments by perfusing a $4 \%$ paraformaldehyde

(26) Lovchik, R. D.; Tonna, N.; Bianco, F.; Matteoli, M.; Delamarche, E. Biomed. Microdevices 2010, 12, 275-282.

(27) Verderio, C.; Matteoli, M. I. Immunol. 2001, 166, 6383-6391.

(28) Bianco, F.; Pravettoni, E.; Colombo, A.; Schenk, U.; Moller, T.; Matteoli, M.; Verderio, C. I. Immunol. 2005, 174, 7268-7277. 


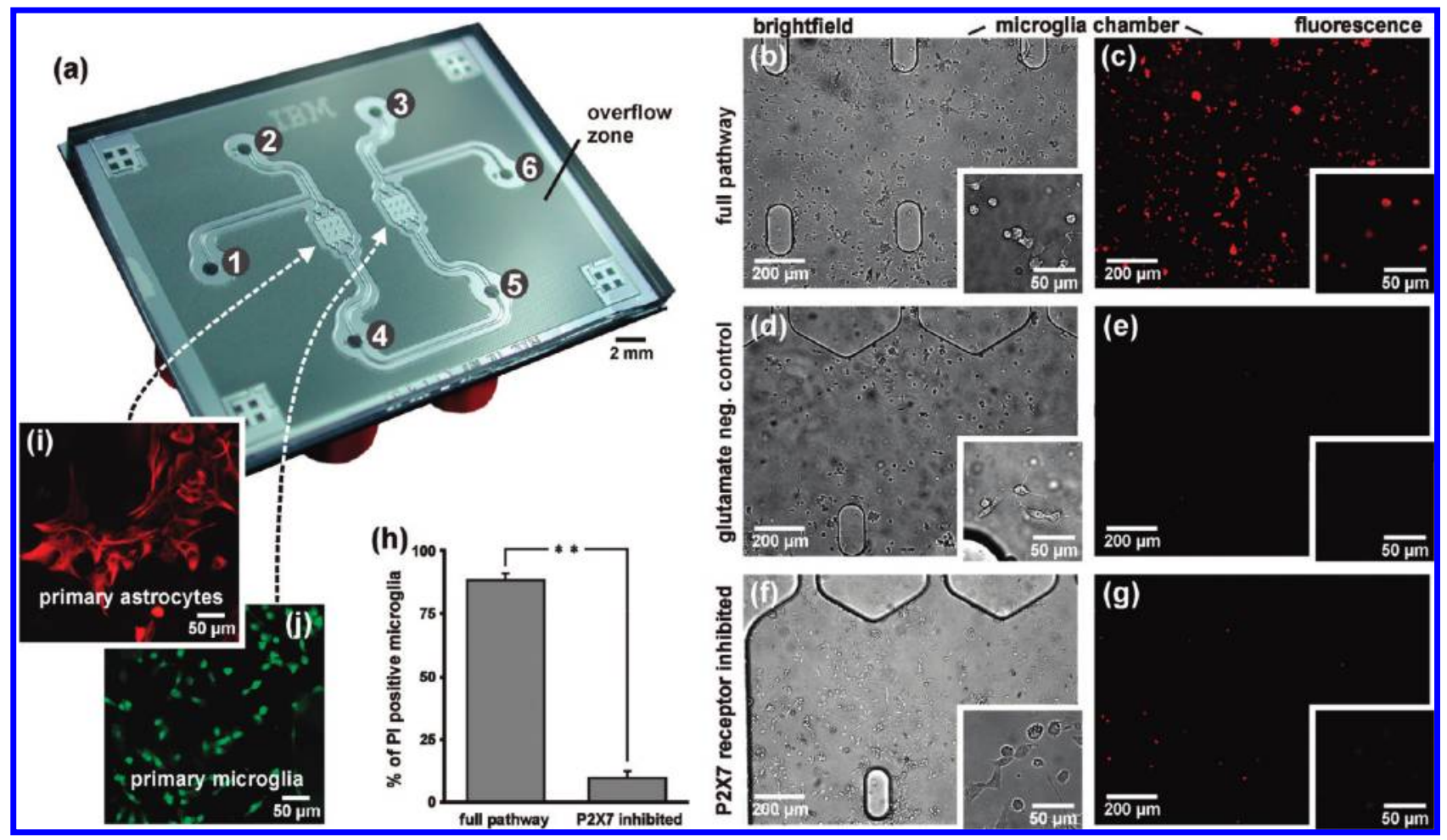

Figure 5. Communication studies between two primary cell populations. (a) Photograph of a two-chamber oMFN sealed with a Si lid (bottom) having 6 ports. Primary astrocytes (left chamber) and microglia (right chamber) were plated and cultured for 3 and 1 DIV, respectively; the lid was then assembled and experiments were performed using several chips. (b, c) Brightfield and corresponding fluorescence microscope images of microglia activated by ATP released from glutamate-stimulated astrocytes in the first chamber; ATP activation of microglia is revealed by the uptake of fluorescent PI (c). (d, e) Control experiment showing the lack of PI uptake by microglia exposed to glutamate in the absence of astrocytes in the first chamber. $(\mathrm{f}, \mathrm{g})$ Control experiment showing that PI uptake by microglia is strongly reduced by concomitant flushing of the P2 $\times 7$ receptor blocker oATP. (h) Quantitation of the percentage of cells labeled by fluorescent PI upon the glutamate-ATP astrocyte-microglia pathway, with and without the P2 $\times 7$ receptor inhibitor oATP in the second chamber. (i, j) Fluorescence microscope images of the primary astrocytes and microglia used in the control in (f) and (g), after the cells were fixed, the lid removed, and the cells stained using dyes for cell specific markers (GFAP for astrocytes, IBA for microglia).

solution for 15 min followed with PBS and, then, further analyzed after removal of the lid. The astrocytes and microglia used in the last experiment (glutamate + oATP, Figure $5 \mathrm{~g}$ ) were stained specifically for either GFAP or IBA after removal of the lid and imaged using a fluorescence microscope, Figure 5i,j.

These experiments are significant because they demonstrate that a pathway between two primary cells can be studied using an oMFN in the presence of, for example, inhibitors of cellular receptors. An obvious application of this method is to carefully screen for neuroprotective action of selected compounds in the context of synaptopathies. For more complex pathways or experiments requiring very precise stimulation of cell populations, flow tracers, such as polystyrene beads, can be added.

\section{CONCLUSIONS}

Primary cultures from the central nervous system, in contrast to cell lines, represent a physiologically more relevant cell model, given that they largely maintain the features of their correlates in situ and, therefore, represent a more reliable system for investigating neuronal development and molecular processes of brain diseases. For this reason, leading neurobiology laboratories worldwide use primary cultures to investigate neuronal development, cell-to-cell signaling, and molecular mechanisms of brain diseases. Also, primary neuronal cultures are increasingly used for preclinical studies on potential drug candidates. ${ }^{29}$ In a recent study, Millet et al. showed that noncross-linked PDMS oligomers affect the viability of primary hippocampal neurons that are plated at low density in a closed microfluidic. ${ }^{15}$ For this reason, culturing cells in an open PDMS chamber brings an additional benefit when working with neurons. Overflow MFNs represent a bridge between the typical workflow in cell biology and the field of microfluidics, as they combine the convenience of working on primary cell cultures with the capabilities of microfluidics for highthroughput, low-volumes, automated, and accurate assays. The possibility of plating cells in a sterile environment, keeping them in standard culturing conditions within incubators, and retrieving them at the right developmental state to use them in a microfluidic device may serve a broad range of purposes, such as performing toxicity, motility, and adhesion assays, as well as cell differentiation studies. We currently are scaling the concept of oMFNs to having three or more interconnected cell chambers, in view of depositing different cell populations in each chamber for dissecting the intercellular flows of information occurring among brain cells. ${ }^{27,28}$ This approach might help

(29) Shaughnessy, L.; Chamblin, B.; McMahon, L.; Nair, A.; Thomas, M. B.; Wakefield, J.; Koentgen, F.; Ramabhadran, R. I. Mol. Neurosci. 2004, 24, 23-32. 
identify the mediators and the cellular sources of molecules involved in neuroinflammation, a process that plays a very active role in the pathophysiology of progressive neurodegenerative disorders, such as Alzheimer's and Parkinson's diseases, and synaptopathies in general.

\section{ACKNOWLEDGMENT}

We thank R. Stutz for his help with the fabrication of the $\mathrm{Si}$ lids and molds for the PDMS oMFNs, L. Gervais and C. Verderio for discussions, and W. Riess and M. Despont for their continuous support. We are grateful to C. Bjornsson and B. Roysam, who provided Figure 1a, and E. Lovchik, who drew Figure 1b. This work was partially supported by CARIPLO No. 2006-0948 to M.M. and by MIUR art. 11 D.M. No. 593/2000 to M.M. and F.B. The research leading to these results has received funding from the European Union Seventh Framework Programme under grant agreement No. HEALTH-F2-2009-241498 (“EUROSPIN" project) to M.M.

Received for review March 25, 2010. Accepted April 3, 2010.

AC100771R 